\title{
Genome-wide association study of normal and atypical progesterone profiles in Holstein-Friesian dairy cows
}

\author{
S. Nyman, ${ }^{*}$ S. I. Duchemin, D. J. de Koning, and B. Berglund \\ Department of Animal Breeding and Genetics, Centre for Reproductive Biology, Swedish University of Agricultural Sciences, \\ SE-750 07 Uppsala, Sweden
}

\section{ABSTRACT}

Female fertility has a major role in dairy production and affects the profitability of dairy cattle. The genetic progress obtained by traditional selection can be slow because of the low heritability of classical fertility traits. Endocrine fertility traits based on progesterone concentration in milk have higher heritability and more directly reflect the cow's own reproductive physiology. The aim of our study was to identify genomic regions for 7 endocrine fertility traits in dairy cows by performing a genome-wide association study with 54,000 SNP. The next step was to fine-map targeted genomic regions with significant SNP using imputed sequences to identify potential candidate genes associated with the normal and atypical progesterone profiles. The association between a SNP and a phenotype was assessed by a single SNP analysis, using a linear mixed model that included a random polygenic effect. Phenotypes and genotypes were available for 1,126 primiparous and multiparous Holstein-Friesian cows from research herds in Ireland, the Netherlands, Sweden, and the United Kingdom. In total, 44 significant SNP associated with 7 endocrine fertility traits were identified on Bos taurus autosome (BTA) 1-4, 6, 8-9, 11-12, 14-17, 19, 21-24, and 29. Three chromosomes, BTA8, BTA17, and BTA23, were imputed from 54,000 SNP genotypes to the whole-genome sequence level with Beagle version 4.1. The fine-mapping identified several significant associations with delayed cyclicity, cessation of cyclicity, commencement of luteal activity, and inter-ovulatory interval. These associations may contribute to an index of markers for genetic improvement of fertility. Several potential candidate genes reported to affect reproduction were also identified in the targeted genomic regions. However, due to high linkage disequilibrium, it

Received July 20, 2018.

Accepted December 18, 2018.

*Corresponding author: sofia.nyman@slu.se was not possible to identify putative causal genes or polymorphisms for any of the regions.

Key words: dairy cattle, fertility, progesterone profile, genome-wide association studies, imputation

\section{INTRODUCTION}

Female fertility has a major role in the dairy production and is an economically important trait affecting profitability in dairy cattle (De Vries, 2006). The genetic progress obtained by traditional selection can be slow, because traits such as interval from calving to first insemination (CFS) often show low heritability, below 5\% (Pryce and Veerkamp, 2001). Low heritability together with an antagonistic relationship between the production and fertility traits complicates genetic improvement for both traits. Endocrine fertility traits, such as progesterone (P4) profiles, might be more useful because they have higher heritabilities and more closely reflect the biology of the cow compared with classical fertility traits. Deviations from a normal estrous cycle, termed atypical P4 profiles, have been associated with compromised fertility (Bulman and Wood, 1980; Royal et al., 2002), and have an antagonistic relationship with milk yield in early lactation (Nyman et al., 2014). Heritabilities of delayed cyclicity and commencement of luteal activity (C-LA) determined by P4 profiles are generally higher. Delayed cyclicity have been reported as having a heritability of 0.24 (Nyman et al., 2014), and C-LA a heritability from 0.12 to 0.18 (Berry et al., 2012; Nyman et al., 2014; Tenghe et al., 2015). A higher heritability estimate might reflect a more effectively estimated genetic variance (Pryce and Veerkamp, 2001). Progesterone-based fertility traits are free from management influences (e.g., estrus detection and insemination dates). Hence, it might be possible to identify genes underlying this genetic variation.

Many genome-wide association studies (GWAS) have been performed on classical fertility traits [e.g., calving to first service (Höglund et al., 2009; Olsen et al., 2011; Berry et al., 2012), pregnancy rates (Berry et al., 2012), and nonreturn rate (Olsen et al., 2011)]. Only 
a few GWAS have been performed on endocrine fertility traits. Berry et al. (2012) found clear associations with C-LA on BTA2 and on BTA21. Furthermore, Tenghe et al. (2016) reported regions on BTA2 and BTA3 (among others) that are likely to include mutations associated with C-LA, interval from C-LA to first insemination, luteal phase length (LPL), inter-ovulatory interval (IOI), and CFS (among other traits). One challenge when working with low heritability traits is that GWAS requires a large number of individuals with phenotype and genotype information (Olsen et al., 2011). Therefore, the high cost associated with data collection in larger population reflects the low number of genomic studies on fertility traits, and especially endocrine fertility traits.

By using whole-genome sequences (WGS), causal variants underlying QTL should be identified more effectively by GWAS as the sequence data are expected to include the causal variants. Daetwyler et al. (2014), who were the first to implement WGS data in cattle, and Druet et al. (2014) found higher precisions in detecting QTL in WGS data. Whole genome sequence data should include the causal variants and have less dependency on population linkage disequilibrium (LD). Furthermore, it is possible to increase the power and precision of QTL mapping by increasing the numbers of markers with imputed sequences (e.g., Höglund et al., 2014; Sahana et al., 2014; Duchemin et al., 2016).

To our knowledge, no previous GWAS have identified genomic regions for the atypical $\mathrm{P} 4$ profiles in dairy cows. The objective of this study was to perform a GWAS to identify genomic regions associated with normal and atypical P4 profiles in Holstein-Friesian (HF) dairy cows. Furthermore, we wanted to fine-map chromosomes with significant SNP using imputed sequences to identify potential candidate genes associated with normal and atypical $\mathrm{P} 4$ profiles.

\section{MATERIALS AND METHODS}

\section{Animals and Phenotypes}

The data consisted of $\mathrm{P} 4$ records from 1,126 primiparous and multiparous HF cows belonging to 4 research herds: (1) Teagasc, Moorepark (Ireland, $\mathrm{n}=168$ ), (2) Jälla, Swedish University of Agricultural Science (Sweden, $\mathrm{n}=224$ ), (3) Scotland's Rural College (United Kingdom, $\mathrm{n}=148$ ), and (4) Wageningen UR Livestock Research (the Netherlands, $\mathrm{n}=666$ ). A more detailed description of the experimental treatments imposed on animals in the different experimental herd, procedures for milk sampling, and P4 analysis have been reported for the Netherlands by Veerkamp et al. (2000), for Ireland by Horan et al. (2005), for Sweden by Petersson et al. (2006), and for the United Kingdom by Pollott and Coffey (2008).

Traits were defined using $\mathrm{P} 4$ records as described in Nyman et al. (2014). Three atypical P4 profiles were analyzed: (1) delayed cyclicity defined as P4 concentrations below the predefined $\mathrm{P} 4$ threshold for luteal phase for more than 56 d postpartum followed by a normal cycle $(\mathrm{n}=178),(2)$ cessation of cyclicity defined as a normal start of cyclicity but interrupted for at least $14 \mathrm{~d}$ with $\mathrm{P} 4$ concentrations below the threshold for luteal phase followed by a normal cycle $(\mathrm{n}=94)$, and (3) prolonged luteal phase defined as a normal start of cyclicity but with high $\mathrm{P} 4$ concentrations (>8 ng/ $\mathrm{mL}$ ) for at least $20 \mathrm{~d}$ followed by a normal cycle $(\mathrm{n}=$ 240). Within each lactation, 4 cycle length traits were analyzed: (1) C-LA, defined as the number of days from calving to the first time the $\mathrm{P} 4$ value crossed the predefined threshold for luteal phase $(\mathrm{n}=1,592)$; $(2)$ IOI, defined as the number of days from the increase in P4 above the predefined threshold for luteal phase in one estrous cycle to the $\mathrm{P} 4$ increase in the next cycle ( $\mathrm{n}=2,068)$; (3) LPL, defined as the number of days from the $\mathrm{P} 4$ increase above the predefined threshold for luteal phase to the time $\mathrm{P} 4$ diminished below the predefined threshold ( $\mathrm{n}=3,376)$; and (4) inter-luteal interval (ILI), defined as the period of time following ovulation in which the corpus luteum secretes $\mathrm{P} 4$ below the predefined $\mathrm{P} 4$ threshold value $(\mathrm{n}=2,122)$.

Milk yield at 60 DIM and the classical fertility traits were also analyzed for comparison with the P4 traits. The classical fertility traits included (1) the interval from calving to first observed heat (CFH), (2) CFS, (3) pregnancy at first service (PFS), (4) the interval from first to last service, and (5) calving interval (CInt).

\section{Genotypes}

The DNA extracted from blood samples was used for genotyping purposes. Genotyping was performed on 1,735 HF cows with the Illumina BovineSNP50 v1 BeadChip (50K; Illumina Inc., San Diego, CA). For each trait, animals were included in the analysis if both genotypic and phenotypic data were available. Therefore, 1,191 phenotypes were available for delayed cyclicity, cessation of cyclicity, and prolonged luteal phase, 793 phenotypes for C-LA and IOI, and 1,068 phenotypes for ILI and LPL.

\section{Significant Associations}

For the 7 fertility traits, a significant association was selected at $10^{-4}$. We wanted to find significant associations for the traits of interest and select the most promising chromosomes for imputation. The $P$-value of $10^{-4}$ 
is considered as a more liberal/less stringent threshold, which could be used to detect associations for complex traits with low heritability and for traits controlled by many genes with small effect (Teyssèdre et al., 2012). A more stringent significance level would reduce the power to validate SNP (Streit et al., 2013), whereas too liberal $P$-values may give high false positive rates.

\section{GWAS on 50K SNP Genotypes}

The quality criteria applied before imputation was a minimum call rate of $95 \%$, a minor allele frequency above $1 \%$, and no extreme deviation from HardyWeinberg equilibrium $(P$-value $>0.01)$. A total of 1,661 genotyped cows and 43,582 markers passed the quality criteria and remained in the study.

All statistical analyses were performed with the GenABEL package RepeatABEL in R 2.15.0 software ( $\mathrm{R}$ Core Team, 2015) using a linear mixed model. Single SNP analyses were performed using the following animal model:

$$
\begin{aligned}
\mathrm{y}_{i j k l m n}=\mu+ & \mathrm{P}_{i}+\mathrm{Y}_{j}+\mathrm{S}_{k}+\mathrm{SNP}_{l}+\mathrm{a}_{m} \\
& +\mathrm{pe}_{n}+\mathrm{e}_{i j k l m n},
\end{aligned}
$$

where $\mathrm{y}_{i j k l m n}$ was the phenotypes for a given trait, $\mu$ was the overall mean, $\mathrm{P}_{i}$ was parity within country, $\mathrm{Y}_{j}$ was calving year within country, $\mathrm{S}_{k}$ was calving season within country, $\mathrm{SNP}_{l}$ was the fixed effect of SNP genotype, $\mathrm{a}_{m}$ was the random polygenic effect of animal, $\mathrm{pe}_{n}$ was the permanent environment to account for repeated measures (i.e., if more than 1 estrous cycle, lactation, or both) within animal, and $\mathrm{e}_{i j k l m n}$ was the random error term. More information about the effects used in the model is provided by Nyman et al. (2014).

After our initial GWAS, we focused on the most promising chromosomes. The chromosomes that showed significant $\left(P<10^{-4}\right)$ associations with several of our 7 traits were considered for further analyses. Then these chromosomes were imputed from the 50K SNP genotypes to sequence level, and a chromosome-wide association study (CWAS) was performed.

\section{Imputations}

A reference population of $547 \mathrm{HF}$ cows and bulls from the sixth run of the 1,000 Bull Genomes Consortium were available (Daetwyler et al., 2014). All positions of the SNP on the 50K SNP genotypes and variants on the sequences were aligned to the bovine genome assembly UMD3.1 (Zimin et al., 2009; SNPchimp, 2017). Alignment, variant calling, and filtering of the sequence data were performed in accordance with Daetwyler et al. (2014).

The reference population was used to impute the genotypes of our experimental population from $50 \mathrm{~K}$ to WGS. This imputation was performed by using Beagle version 4.1 (Browning and Browning, 2016). Imputation started by checking the inconsistency between the 50K SNP genotypes and the WGS reference population of $547 \mathrm{HF}$ bulls, using the Conform-gt software (http: //faculty.washington.edu/browning/conform-gt.html). After this check, the cows were imputed from $50 \mathrm{~K}$ to sequence level. The imputed sequences were filtered to remove poorly imputed genotypes. The accuracy of imputation (AR2) of each marker was provided by Beagle as the bi-allelic $r^{2}$. A variant was considered as well imputed and retained for further analyses if the average accuracy of imputation was equal to or higher than 0.7 .

\section{CWAS on Imputed Sequences}

The CWAS with imputed sequence data for the most promising regions was run by using the same statistical model as in the GWAS. Two CWAS were run: (1) where only variants imputed with an AR2 $\geq 0.2$ were included and (2) where only variants imputed with an AR2 $\geq 0.7$ were included. Single-marker analyses were run using the same model as for the GWAS. The genomic relationship matrix was based on the 50K SNP genotypes, as in the GWAS. The WGS genotypes used in the imputation were not included in the genomic relationship matrix in the CWAS.

Only results for the 50K SNP genotypes and the sequence data with polymorphic markers with an AR2 $\geq$ 0.7 will be shown.

\section{Candidate Genes}

Based on the CWAS results for each trait, putative candidate genes were identified based on the Bos taurus UMD3.1 genome assembly (Zimin et al., 2009; http://bovinegenome.org/). The gene information was based on the Ensembl database (version 91, accessed February 2018 at http://www.ensembl.org/) and the information about the function of the genes was based on the GeneCards human gene database (accessed February 2018 at http://www.genecards.org/).

\section{RESULTS}

\section{GWAS on 50K SNP Genotypes}

Significant SNP for the analyzed endocrine fertility traits are presented in Table 1 . In total, 44 significant 
SNP associated with endocrine fertility traits were detected on BTA1-4, 6, 8-9, 11-12, 14-17, 19, 21-24, and 29. Regarding the 50K SNP genotypes, the strongest association was found on BTA9 with a $-\log _{10}(P$-value $)$ of 6.30 for cessation of cyclicity (Table 1 ).

Twenty-four significant SNP associated with milk yield and the classical fertility traits were identified on BTA1, 2, 4, 5, 8, 10-12, 14-15, 19, 25, and 28 (Supplemental Table S1; https://doi.org/10.3168/jds.2018 -15418). The strongest SNP associated with classical fertility traits was identified for CInt on BTA14 and significant at a $-\log _{10}(P$-value) of 5.59 (Supplemental Table S1; https://doi.org/10.3168/jds.2018-15418).

Because SNP on BTA8, BTA17, and BTA23 showed significant associations with 4 of the studied endocrine fertility traits, we focused on these chromosomes for the CWAS. Moreover, on BTA8 we identified significant SNP for the classical fertility traits CFH and CFS.

Manhattan plots for the atypical P4 profiles and cycle length traits for the 50K SNP genotypes are presented in Supplemental Figures S1 and S2, respectively (https: //doi.org/10.3168/jds.2018-15418). Quantile-quantile plots for the atypical P4 profiles and for the cycle length traits are presented in Supplemental Figures S3 and S4, respectively (https://doi.org/10.3168/jds.2018-15418).

\section{Imputation to WGS on BTA8, BTA17, and BTA23}

Table 2 presents information about the SNP and the imputed sequences on BTA8, BTA17, and BTA23. After imputation with an AR2 $\geq 0.7$, the total number of variants on BTA8 increased from 1,962 SNP on the $50 \mathrm{~K}$ genotypes to 23,051 variants, on BTA17 from 1,338 SNP to 26,901 variants, and on BTA23 from 914 SNP to 21,376 variants. This is an increase of 12,20 , and 23 times for BTA8, BTA17, and BTA23, respectively. The average accuracy of imputation using our approach was 0.87 for BTA8, 0.86 for BTA17, and 0.86 for BTA23.

\section{CWAS for Imputed Sequences on BTA8, BTA17, and BTA23}

We ran a CWAS based on imputed sequences for BTA8, BTA17, and BTA23. Regarding imputed sequences on BTA8, 9 significant variants were associated with cessation of cyclicity, 6 with delayed cyclicity, and 2 with C-LA (Supplemental Table S2; https://doi .org/10.3168/jds.2018-15418). For CFS, we identified 57 significant variants on BTA8 (details not given). Regarding imputed sequences on BTA17, 10 significant variants were associated with cessation of cyclicity, 5 with IOI, and 20 with delayed cyclicity (Supplemental Table S3; https://doi.org/10.3168/jds.2018-15418).
We identified 5 significant variants for $\mathrm{CFH}$ and 67 for CFS on BTA17 (details not given). Regarding imputed sequences on BTA23, 2 significant variants were associated with C-LA, 4 with delayed cyclicity, and 1 with cessation of cyclicity (Supplemental Table S4; https://doi.org/10.3168/jds.2018-15418). For CFS, we identified 11 significant variants on BTA23 (details not given). Figures 1 and 2 show Manhattan plots for BTA8, BTA17, and BTA23 with the 50K SNP genotypes overlaid with the imputed variants $(\mathrm{AR} 2 \geq 0.7)$.

For delayed cyclicity, we detected 2 QTL regions on BTA8 (between 38.9 and $40.9 \mathrm{Mbp}$, and between 58.9 and $60.9 \mathrm{Mbp}$ ), 1 on BTA17 (between 52.8 and 55.2 $\mathrm{Mbp}$ ), and 2 QTL regions on BTA23 (between 29.90 and 31.90, and between 41.2 and 43.2 Mbp; Figure 1). For cessation of cyclicity, 1 significant variant was identified on BTA8 (at 36.22 Mbp), 1 on BTA17 (at 47.01 Mbp), and 1 on BTA23 (at $7.91 \mathrm{Mbp}$; Figure 1). For C-LA, 1 QTL region was detected on BTA8 (between 58.9 and $60.9 \mathrm{Mbp}$ ), 1 on BTA17 (between 52.8 and $55.2 \mathrm{Mbp}$ ), and 1 on BTA23 (between 41.2 and 43.2 Mbp; Figure 2). For IOI, we detected 1 QTL region on BTA17 (between 52.8 and 55.2 Mbp; Figure 2). Manhattan plots for delayed cyclicity, cessation of cyclicity, C-LA, and IOI with the imputed variants with AR2 $\geq 0.7$ on BTA8, BTA17, and BTA23 are presented in Supplemental Figure S5 (https://doi.org/10.3168/jds .2018-15418).

\section{Overlapping Regions}

Delayed cyclicity and C-LA had overlapping regions with significant and suggestive variants in all 5 of the detected QTL regions (Figure 3). On BTA17 the observed QTL region included significant variants associated with delayed cyclicity, C-LA (close to significance), and IOI (Figure 3).

No significant variants or overlapping regions were identified for the endocrine fertility traits, $\mathrm{CFH}$ and CFS (results not presented). Using the 50K SNP genotypes, we identified overlapping regions with significant SNP for cessation of cyclicity and CInt on BTA14, and for ILI and milk yield on BTA11.

\section{Potential Candidate Genes}

We used a window of 1 Mbp upstream and downstream of a significant variant for checking candidate genes on the chromosomes, except for a region on BTA17 where we used a wider window $(2.4 \mathrm{Mbp})$ to include all significant variants. The 2 QTL regions on $\mathrm{BTA} 8$, the 1 region on BTA17, and the 2 regions on BTA23 were checked for possibly candidate genes. We 
Table 2. Number of SNP at the 54K SNP genotypes and the number of imputed sequences for all imputed variants (all), with an imputed accuracy $(\mathrm{AR} 2) \geq 0.2$ and with an AR2 $\geq 0.7$, and the number of indels on BTA8, BTA17, and BTA23

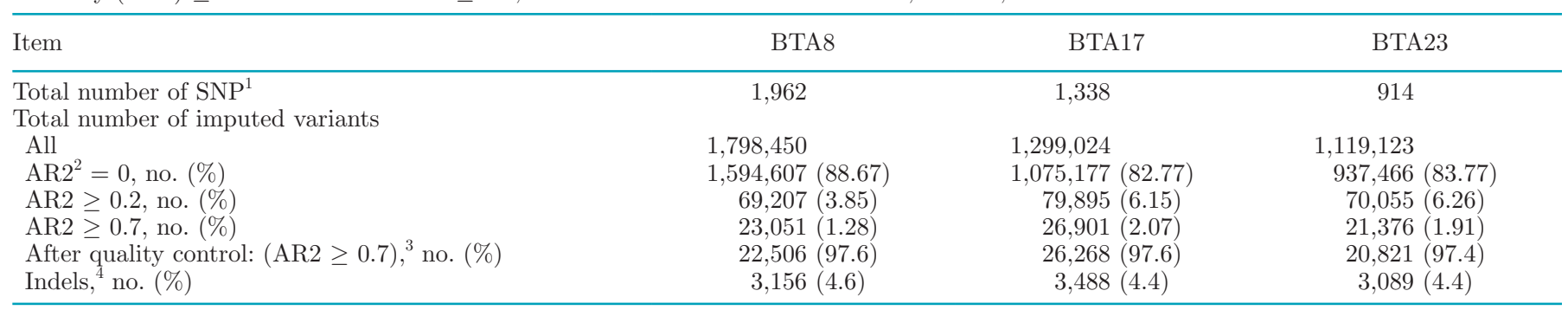

${ }^{1}$ SNP from the Illumina BovineSNP50 v1 BeadChip (Illumina Inc., San Diego, CA).

${ }^{2} \mathrm{AR} 2=$ imputation accuracy.

${ }^{3}$ Number and percentage of imputed variants (AR2 $\left.\geq 0.7\right)$ after quality control.

${ }^{4}$ Number and percentage of indels out of all imputed variants with an AR2 $\geq 0.2$.

identified many genes in these 5 regions, but as presented in Table 3 we only considered the genes affecting reproduction (i.e., the reproduction system, embryo phenotype, hormones affecting fertility, and endocrine morphology/physiology). On BTA8 we identified 4 genes between 38.9 and $40.9 \mathrm{Mbp}$ and 5 genes between 58.9 and $60.9 \mathrm{Mbp}$. In the targeted genomic region on BTA17, we identified 9 genes. On BTA23 we identified 2 genes between 29.90 and $31.90 \mathrm{Mbp}$, and 3 genes between 41.2 and $43.2 \mathrm{Mbp}$.

\section{DISCUSSION}

In this study, we performed a GWAS for 7 endocrine fertility traits, milk yield at d 60 DIM, and 5 classical fertility traits. When using imputed sequences with high imputation accuracy, GWAS can assist in fine-mapping and increase the chance of identifying a causal variant (Marchini and Howie, 2010). The number of variants increased at least 10 times, after the imputation from $50 \mathrm{~K}$ to sequence data. With the imputed sequences, we identified potential candidate genes within 5 observed QTL regions at 3 chromosomes, which contained the significant top variants for each trait. The phenotypic and the genetic variance explained by the markers were estimated for the top SNP on the 50K genotypes (Table 1) and for the 5 QTL regions on BTA8, BTA17, and BTA23 (Supplemental Tables S2, S3, and S4; https: //doi.org/10.3168/jds.2018-15418). For all the studied traits, the phenotypic variance explained by the markers was low. The genetic variance explained by the markers ranged between 5 and $80 \%$. This may be an overestimation because the QTL variance, as illustrated by Ekine et al. (2014), is affecting both the genetic variance and the residual variance.

To our knowledge, this is the first GWAS detecting genomic regions associated with the atypical P4 profiles. To date, no QTL region has been reported for C-LA on BTA8, BTA17, and BTA23. Earlier studies have reported significant associations with C-LA on other chromosomes; for example, Berry et al. (2012) and Tenghe et al. (2016) detected a QTL region associated with C-LA on BTA2. Although we used different methods, we found the same significant SNP on BTA2 in this study as was found by Berry et al. (2012). The inclusion of this SNP in genetic evaluations might be of interest, thus, it deserves further investigation. Berry et al. (2012) also reported a possible QTL for C-LA at position $9.38 \mathrm{Mbp}$ on BTA21. In the present study, no significant SNP on BTA21 was associated with C-LA. However, on BTA21 we found a significant SNP associated with IOI at $8.78 \mathrm{Mbp}$ with a $-\log _{10}(P$-value $)$ of 4.45. Berry et al. (2012) also found significant SNP associated with $\mathrm{CFH}$ on BTA8, but this was not at the same position as in the present study. Tenghe et al. (2016) reported significant SNP associated with LPL on BTA3 and BTA17, whereas in the present study we found interesting regions for IOI on the same chromosomes. As IOI combines information from LPL and ILI, this may suggest that the same genes influence both IOI and LPL. Nevertheless, on BTA3 there was a difference of $10 \mathrm{Mbp}$ between these $2 \mathrm{SNP}$, and on BTA17, the distance was even greater. Discrepancies between sample size, significance levels, methods of analysis, and number of observations could explain the differences between the results of Berry et al. (2012), Tenghe et al. (2016), and our study.

Results from the GWAS of the 50K SNP genotypes showed significant SNP on BTA1 with an overlapping region for cessation of cyclicity (at $116.35 \mathrm{Mbp}$ ) and PFS (at $118.71 \mathrm{Mbp}$ ). The genetic correlation between cessation of cyclicity and PFS could not be estimated in Nyman et al. (2014) because of the low genetic variation of cessation of cyclicity. On BTA11, we found 
Delayed cyclicity
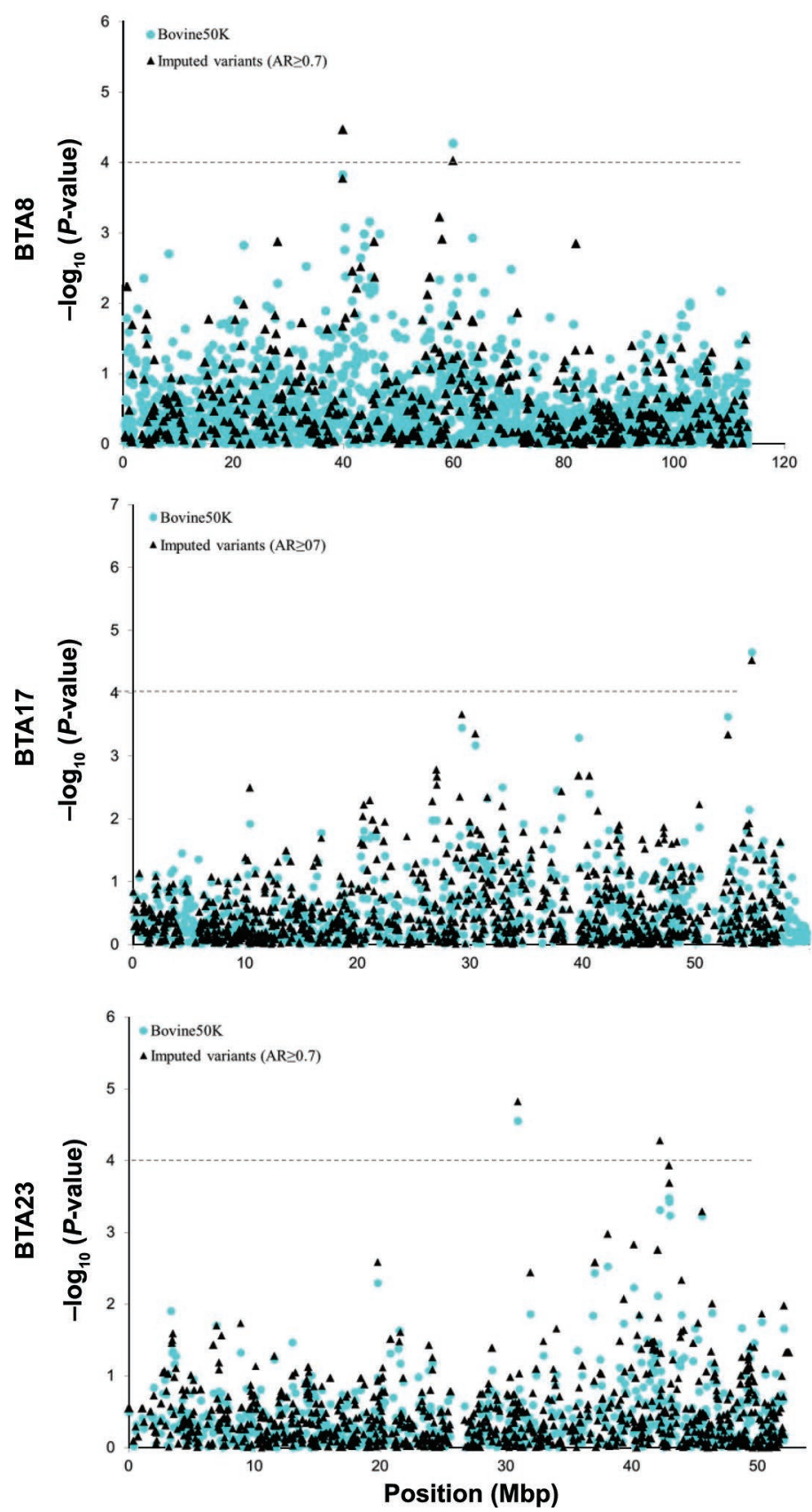

Cessation of cyclicity
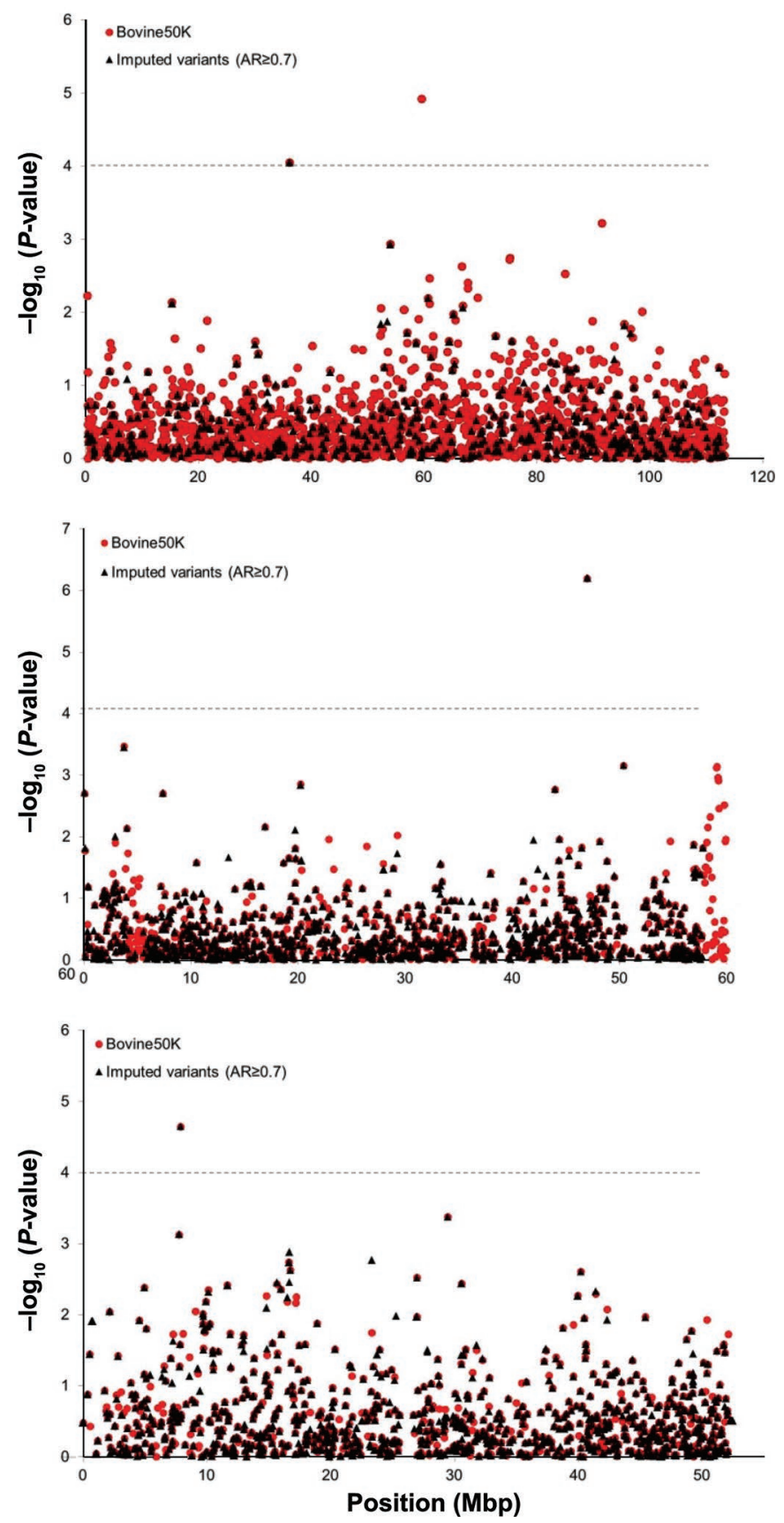

Figure 1. Chromosome-wide association study for delayed cyclicity (turquoise) and cessation of cyclicity (red) on BTA8, BTA17, and BTA23. Bovine 50K SNP genotypes (turquoise and red circles) overlaid with imputed variants with an imputation accuracy (AR2) $\geq 0.7$ (black triangles). The black dotted line is the genome-wide significance level of $-\log _{10}(P$-value $) \geq 4$ based on $50 \mathrm{~K}$ SNP genotypes.

a significant SNP associated with ILI at $54.70 \mathrm{Mbp}$ and with milk yield at 60 DIM at $54.37 \mathrm{Mbp}$. In the study by Nyman et al. (2014), the genetic correlation between ILI and milk yield at 60 DIM was low (0.25) and unfavorable. Even though there was a low correlation, the genes affecting milk yield and ILI were located close to each other. This finding suggests that breeding for higher milk yield may influence ILI, resulting in a longer period of low P4 after ovulation.

We detected many possible candidate genes in the 5 observed QTL regions on BTA8, BTA17, and BTA23. Only genes reported to affect reproduction (e.g., embry- 
C-LA

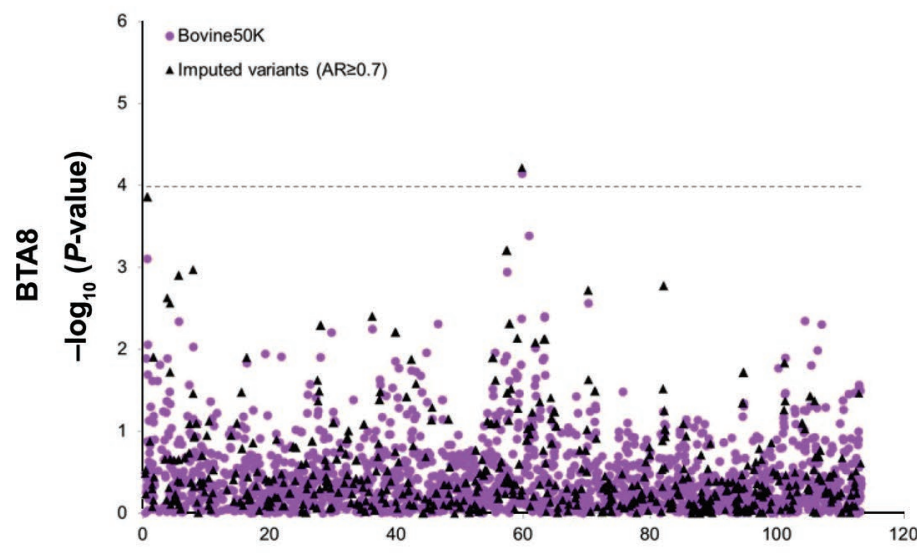

IOI
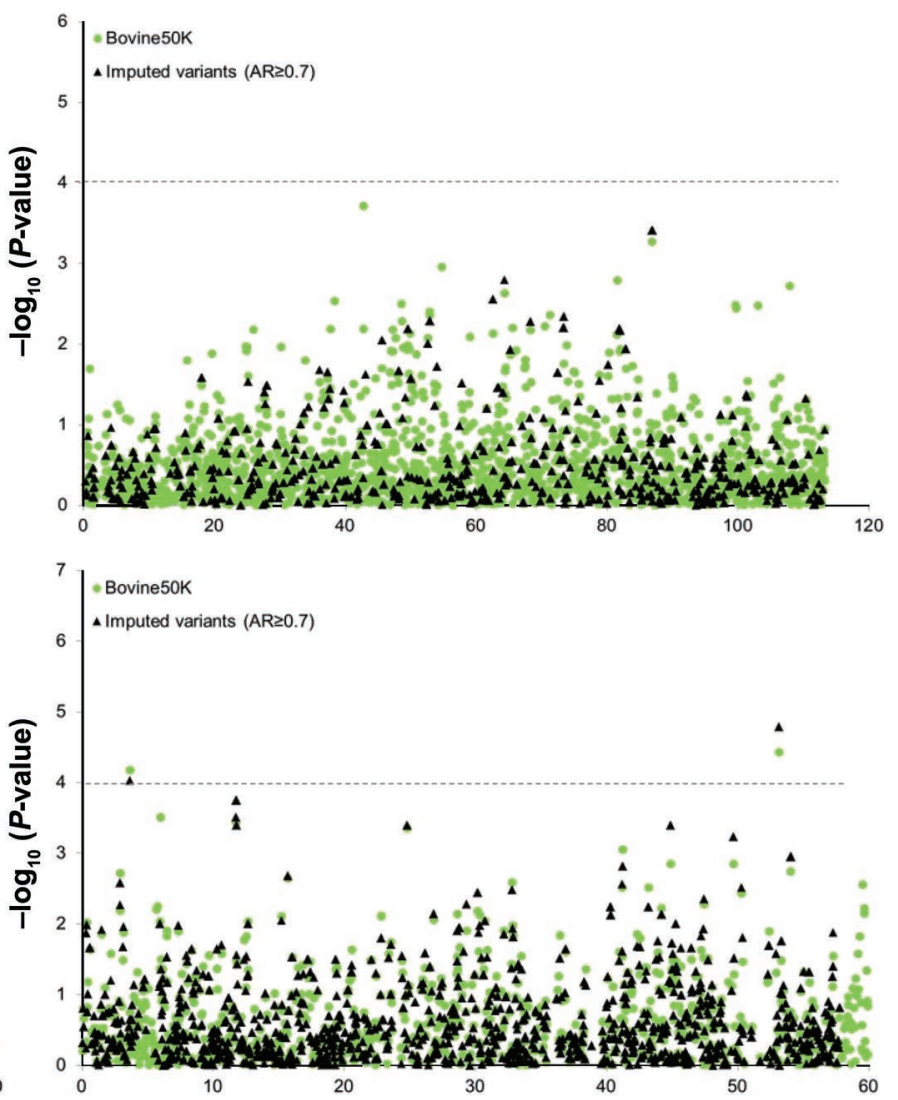
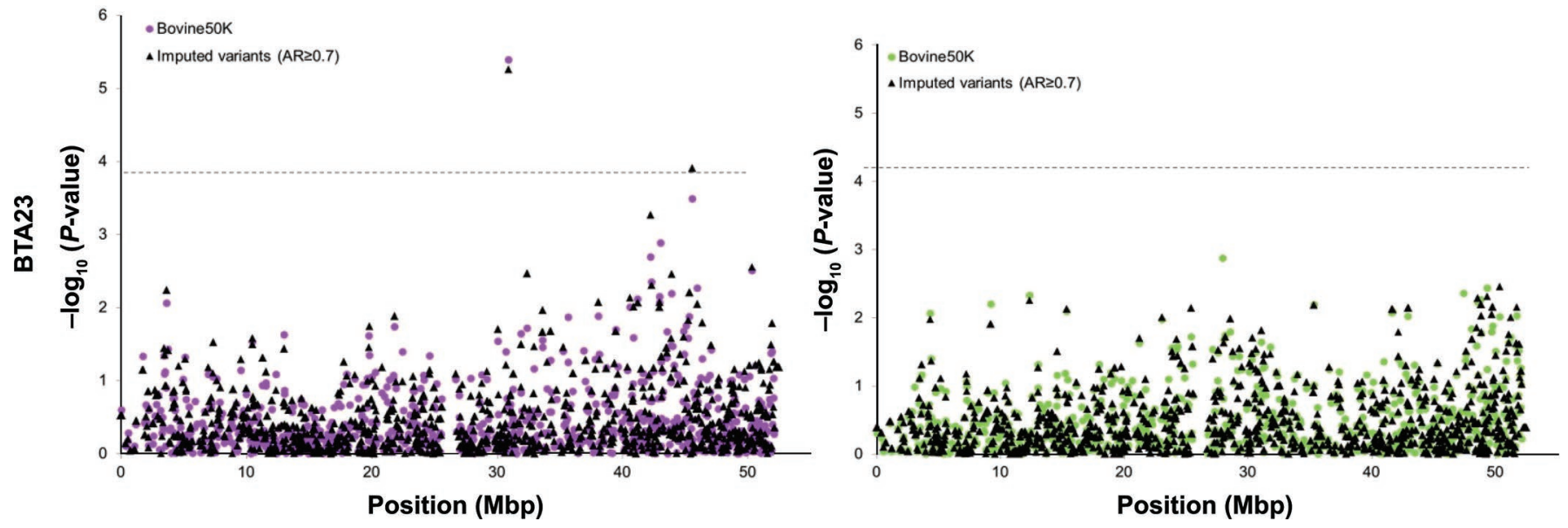

Figure 2. Chromosome-wide association study for commencement of luteal activity (C-LA; purple) and inter-ovulatory interval (IOI; green) on BTA8, BTA17, and BTA23. Bovine 50K SNP genotypes (purple and green circles) overlaid with imputed variants with an imputation accuracy $(\mathrm{AR} 2) \geq 0.7$ (black triangles). The black dotted line is the genome-wide significance level of $-\log _{10}(P$-value $) \geq 4$ based on $50 \mathrm{~K}$ SNP genotypes.

onic and reproductive phenotypes, abnormalities in the reproductive system, and morphology and physiology of the endocrine gland) were considered in this study. Due to high LD between the imputed variants (Figure 3), it was not possible to specify genes or polymorphisms as causal factors for any of the regions. Compared with humans, long-range LD blocks are more common in dairy cattle, especially in HF cows. This is due to the intensive use of relatively few sires for breeding the next generation, which results in a relatively small effective population size (Schmid and Bennewitz, 2017).

On BTA 8 we identified 9 possible candidate genes controlling fertility functions (Table 3). Earlier studies have also reported genes on BTA 8 that are controlling 
fertility (e.g., Peters et al., 2013). Peters et al. (2013) identified genes affecting conception rate at first calving in Brangus cattle, and Daetwyler et al. (2014) identified a mutation in the SMC2 gene when studying animals from the 1,000 Bull Genomes Project. The SMC2 mutation is reported to cause embryonic loss. Kühn et al. (2003) identified genes affecting dystocia and stillbirths in Holstein cattle on BTA8. In the present study, we identified 9 interesting genes controlling fertility on BTA17. Frischknecht et al. (2018) found a QTL region for nonreturn rate at $\mathrm{d} 56$, and the interval from first to last insemination on BTA8 in Brown Swiss cattle. Liu et al. (2017) reported strong associations with conception rate at first insemination and the interval to first insemination in Chinese and Nordic Holstein cows on BTA23 and for heifers on BTA17. In the present study, we identified 5 interesting genes in the 2 QTL regions on BTA23. Blattman et al. (1996) identified genes in a QTL region on BTA23 affecting ovulation rate and Cole et al. (2011) found significant SNP associated with daughter stillbirth. Few reports have studied endocrine fertility traits, although Tenghe et al. (2016) identified significant SNP on BTA8, BTA17, and BTA23 for proportion luteal activity between d 25 and 60 postpartum, first LPL, interval from C-LA to first service, and CFS. All reports with the potential genes on BTA8, BTA17, and BTA23 suggest that these 3 chromosomes are of interest when looking for genomic markers to improve the genetic evaluation for fertility.

Fertility is complex, composed of many sub-traits, and affected by many genes. In the present study, we identified significant SNP spread over the chromosomes, with some exceptions. We identified significant SNP at different locations associated with the same
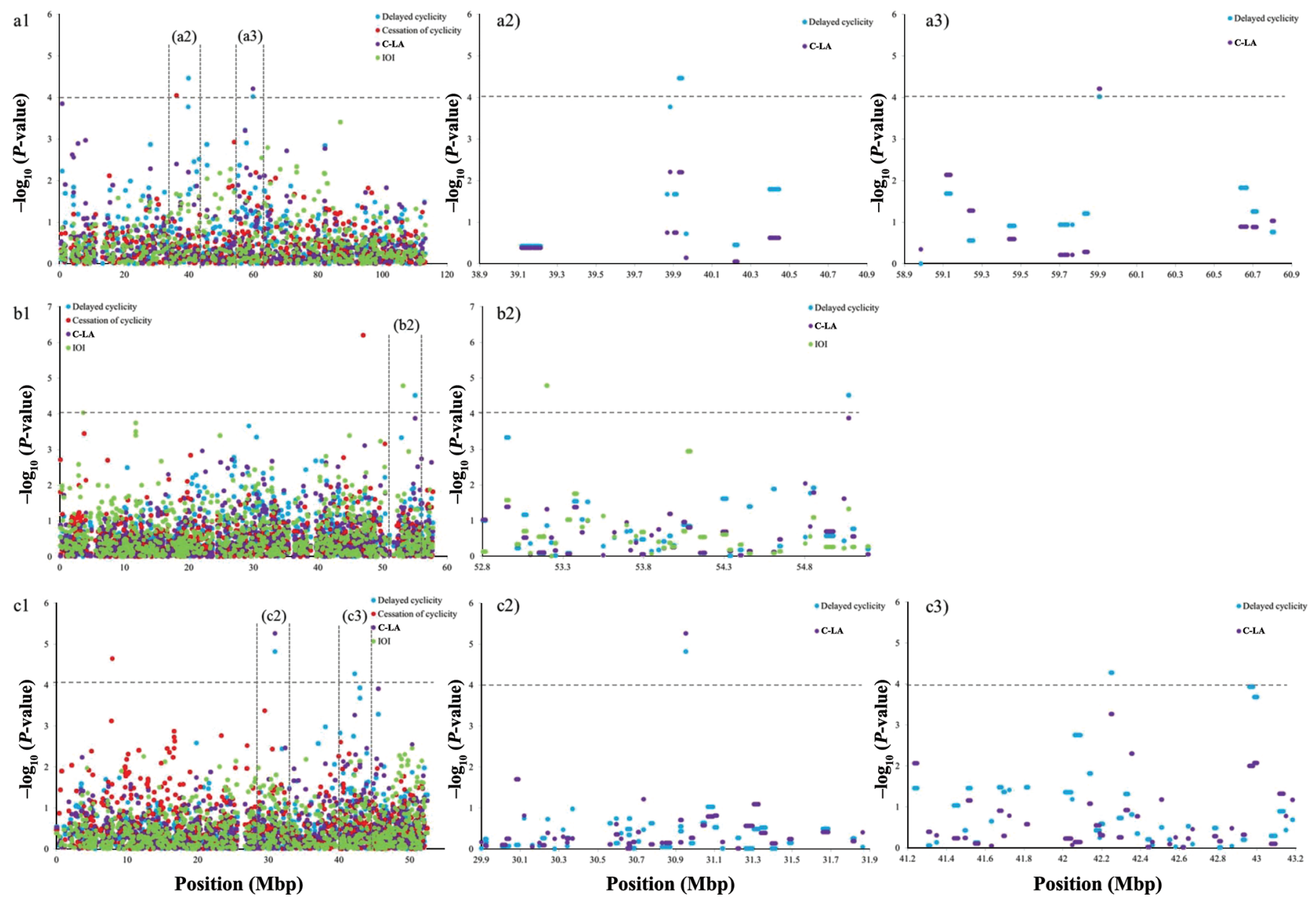

Figure 3. Fine-mapping [imputed sequences with an imputation accuracy (AR2) $\geq 0.7$ ] of (a1) BTA8, (b1) BTA17, and (c1) BTA23 showing an overlay of delayed cyclicity, cessation of cyclicity, commencement of luteal activity (C-LA), and inter-ovulatory interval (IOI). Fine-mapping of delayed cyclicity and C-LA between 38.9 and $40.9 \mathrm{Mbp}$ (a2) and 58.9 and $60.9 \mathrm{Mbp}$ (a3) on BTA8; of delayed cyclicity, C-LA, and IOI between 52.8 and 55.2 Mbp (b2) on BTA17; and of delayed cyclicity and C-LA between 29.9 and 31.9 Mbp (c2) and 41.2 and 43.2 Mbp (c3) on BTA23. The black dotted line is the genome-wide significance level of $-\log _{10}(P$-value $) \geq 4$ based on $50 \mathrm{~K}$ SNP genotypes. 
Table 3. Position of top variant on BTA8, BTA17, and BTA23, possible candidate genes, and start and end position of these genes

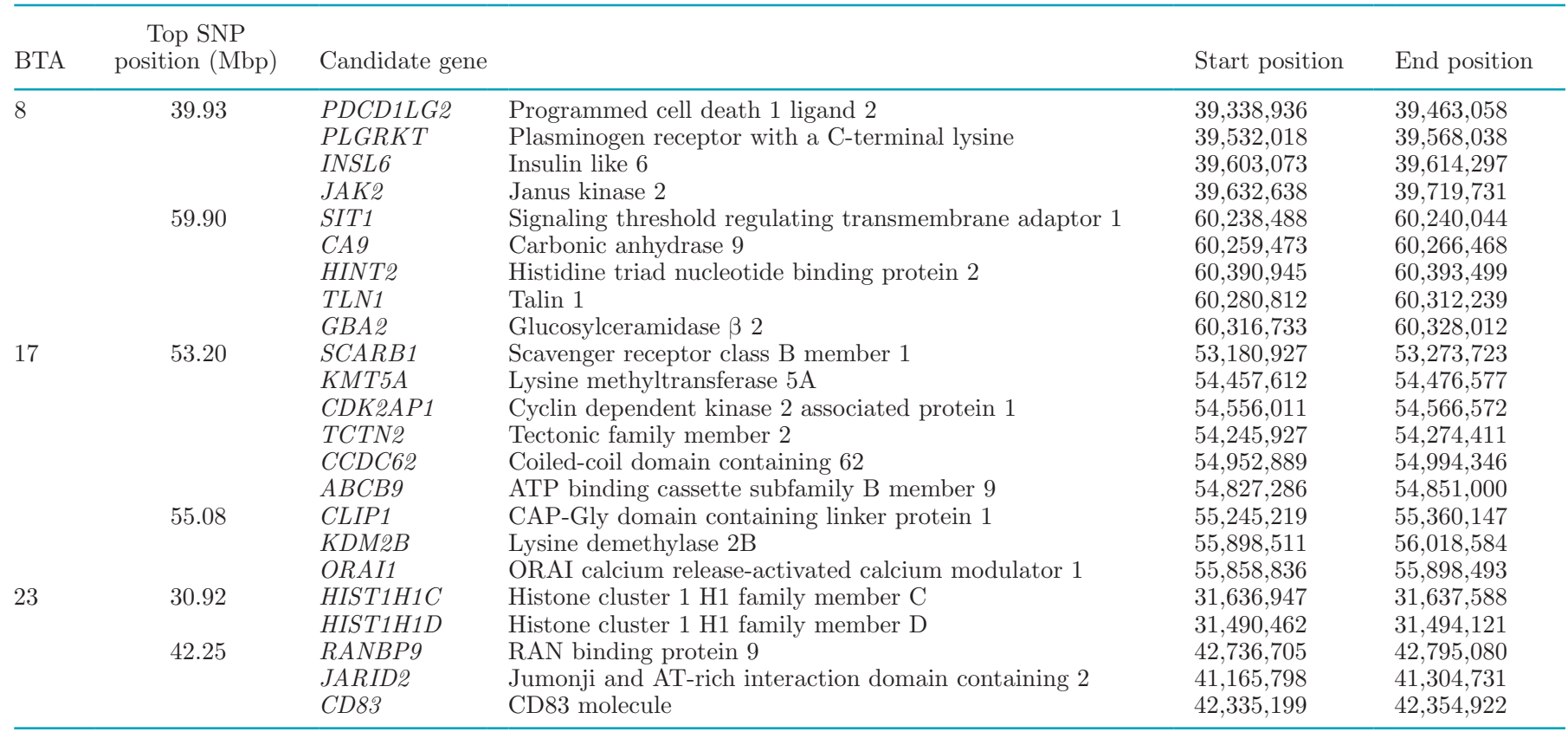

traits. For example, delayed cyclicity was associated with significant SNP and variants on BTA8, BTA17, and BTA23, but also on BTA16 (only analyzed with the 50K SNP genotypes; Table 1). Genes with pleiotropic effect are genes that affect more than one trait. In our study we detected several QTL regions with the same significant SNP or variant associated with both delayed cyclicity and C-LA [e.g., SNP "ARS-BFGLNGS-27920" on BTA17 (Table 1), a variant positioned at $30,953,561 \mathrm{bp}$, and a variant positioned at 30,954,230 bp on BTA23 (Supplemental Table S4; https://doi.org/ 10.3168/jds.2018-15418)]. This suggests that the same genes may control these traits. On the other hand, we detected other regions with variants that were only associated with one of the traits. The complexity of the traits manifested with the absence of very strong associations for either of the traits suggests that many genes with small affects are involved, which makes it difficult to identify causal genes. Further exploration of the genetic basis for endocrine fertility traits would require a much larger GWAS study in terms of many genotyped animals with $\mathrm{P} 4$ records. The increasing use of in-line data is one possibility. HerdNavigator (DeLaval, Tumba, Sweden) is an on-farm milk analysis tool collecting large amounts of phenotypic data. Combined with genotyping of the cows, a much larger GWAS using the same approach could be feasible in the future and be useful to validate the results in this study.

The GWAS results can increase the biological knowledge about traits, which could be of interest in the breeding evaluation (Goddard et al., 2016) and provide information on the genetic architecture of the quantitative trait.

\section{CONCLUSIONS}

We detected a large number of potential chromosomal regions for the observed fertility traits. The fine-mapping of QTL regions on BTA8, BTA17, and BTA23, using imputed sequence variants, identified several significant associations with delayed cyclicity and C-LA that can contribute to an index of markers for genetic improvement of fertility. In the observed QTL regions, we identified several potential candidate genes reported to affect reproduction. However, due to high LD, it was not possible to specify putative causal genes or polymorphisms for any of the regions.

\section{ACKNOWLEDGMENTS}

The authors acknowledge the RobustMilk project for providing data. We also acknowledge Lars Rönnegård (Swedish University of Agricultural Sciences, Uppsala) for his expertise and help with our work with RepeatABEL. The Swedish contribution to the 1,000 Bull Genomes Project was supported by the Mistra Biotech project, a research program financed by Mistra-the Swedish foundation for strategic environmental research (Stockholm) and Swedish University of Agricultural Sciences (Uppsala). 


\section{REFERENCES}

Berry, D. P., J. W. M. Bastiaansen, R. F. Veerkamp, S. Wijga, E. Wall, B. Berglund, and M. P. L. Calus. 2012. Genome-wide association for fertility traits in Holstein-Friesian dairy cows using data from experimental research herds in four European countries. Animal 6:1206-1215. https://doi.org/10.1017/S1751731112000067.

Blattman, A. N., B. W. Kirkpatrick, and K. E. Gregory. 1996. A search for quantitative trait loci for ovulation rate in cattle. Anim. Genet. 27:157-162. https://doi.org/10.1111/j.1365-2052.1996.tb00943.x.

Browning, B. L., and S. R. Browning. 2016. Genotype imputation with millions of reference samples. Am. J. Hum. Genet. 98:116-126. https://doi.org/10.1016/j.ajhg.2015.11.020.

Bulman, D. C., and P. D. P. Wood. 1980. Abnormal patterns of ovarian activity in dairy cows and their relationship with reproductive performance. Anim. Prod. 30:177-188.

Cole, J. B., G. R. Wiggans, L. Ma, T. S. Sonstegard, T. J. Lawlor Jr., B. A. Crooker, C. P. van Tassell, J. Yang, S. Wang, L. K. Matukumalli, and Y. Da. 2011. Genome-wide association analysis of thirty one production, health, reproduction and body conformation traits in contemporary U.S. Holstein cows. BMC Genomics 12:408. https://doi.org/10.1186/1471-2164-12-408.

Daetwyler, H. D., A. Capitan, H. Pausch, P. Stothard, R. van Binsbergen, R. F. Brondum, X. Liao, A. Djari, S. C. Rodriguez, C. Grohs, D. Esquerre, O. Bouchez, M. N. Rossignol, C. Klopp, D. Rocha, S. Fritz, A. Eggen, P. J. Bowman, D. Coote, A. J. Chamberlain, C. Anderson, C. P. Van Tassell, I. Hulsegge, M. E. Goddard, B. Guldbrandtsen, M. S. Lund, R. F. Veerkamp, D. A. Boichard, R. Fries, and B. J. Hayes. 2014. Whole-genome sequencing of 234 bulls facilitates mapping of monogenic and complex traits in cattle. Nat. Genet. 46:858-865. https://doi.org/10.1038/ng.3034.

De Vries, A. 2006. Economic value of pregnancy in dairy cattle. J. Dairy Sci. 89:3876-3885. https://doi.org/10.3168/jds.S0022 $-0302(06) 72430-4$

Druet, T., I. M. Macleod, and B. J. Hayes. 2014. Toward genomic prediction from whole-genome sequence data: Impact of sequencing design on genotype imputation and accuracy of predictions. Heredity 112:39-47. https://doi.org/10.1038/hdy.2013.13.

Duchemin, S. I., M. Glantz, D. J. de Koning, M. Paulsson, and W. F. Fikse. 2016. Identification of QTL on chromosome 18 associated with non-coagulating milk in Swedish Red cows. Front. Genet. 7:57. https://doi.org/10.3389/fgene.2016.00057.

Ekine, C. C., S. J. Rowe, S. C. Bishop, and D. J. de Koning. 2014. Why breeding values estimated using familial data should not be used for genome-wide association studies. G3 (Bethesda) 4:341-347.

Frischknecht, M., T. H. E. Meuwissen, B. Bapst, F. R. Seefried, C. Flury, D. Garrick, H. Signer-Hasler, C. Stricker, Intergenomics Consortium, A. Bieber, R. Fries, I. Russ, J. Sölkner, A. Bagnato, and B. Gredler-Grandl. 2018. Short communication: Genomic prediction using imputed whole-genome sequence variants in Brown Swiss cattle. J. Dairy Sci. 101:1292-1296. https://doi.org/10.3168/ jds.2017-12890.

Goddard, M. E., K. E. Kemper, I. M. MacLeod, A. J. Chamberlain, and B. J. Hayes. 2016. Genetics of complex traits: prediction of phenotype, identification of causal polymorphisms and genetic architecture. Proc. Biol. Sci. 283:20160569. https://doi.org/10.1098/ rspb.2016.0569.

Höglund, J. K., A. J. Buitenhuis, B. Guldbrandtsen, G. Su, B. Thomsen, and M. S. Lund. 2009. Overlapping chromosomal regions for fertility traits and production traits in the Danish Holstein population. J. Dairy Sci. 92:5712-5719. https://doi.org/10.3168/jds.2008 -1964 .

Höglund, J. K., G. Sahana, B. Guldbrandtsen, and M. S. Lund. 2014. Validation of associations for female fertility traits in Nordic Holstein, Nordic Red and Jersey dairy cattle. BMC Genet. 15:8. https: //doi.org/10.1186/1471-2156-15-8.

Horan, B., J. F. Mee, P. O'Connor, M. Rath, and P. Dillon. 2005. The effect of strain of Holstein-Friesian cow and feeding system on postpartum ovarian function, animal production and conception rate to first service. Theriogenology 63:950-971. https://doi.org/ 10.1016/j.theriogenology.2004.05.014.

Kühn, Ch., J. Bennewitz, N. Reinsch, N. Xu, H. Thomsen, C. Looft, G. A. Brockmann, M. Schwerin, C. Weimann, S. Hiendleder, G. Erhardt, I. Medjugorac, M. Förster, B. Brenig, F. Reinhardt, R. Reents, I. Russ, G. Averdunk, J. Blümel, and E. Kalm. 2003. Quantitative trait loci mapping of functional traits in the German Holstein cattle population. J. Dairy Sci. 86:360-368. https://doi .org/10.3168/jds.S0022-0302(03)73614-5.

Liu, A., Y. Wang, G. Sahana, Q. Zhang, L. Liu, M. Sandø Lund, and G. Su. 2017. Genome-wide association studies for female fertility traits in Chinese and Nordic Holsteins. Sci. Rep. 7:8487. https:// doi.org/10.1038/s41598-017-09170-9.

Marchini, J., and B. Howie. 2010. Genotype imputation for genomewide association studies. Nat. Rev. Genet. 11:499-511. https://doi .org/10.1038/nrg2796.

Nyman, S., K. Johansson, D. J. de Koning, D. P. Berry, R. F. Veerkamp, E. Wall, and B. Berglund. 2014. Genetic analysis of atypical progesterone profiles in Holstein-Friesian cows from experimental research herds. J. Dairy Sci. 97:7230-7239. https://doi .org/10.3168/jds.2014-7984.

Olsen, H. G., B. J. Hayes, M. P. Kent, T. Nome, M. Svendsen, A. G. Larsgard, and S. Lien. 2011. Genome-wide association mapping in Norwegian Red cattle identifies quantitative trait loci for fertility and milk production on BTA12. Anim. Genet. 42:466-474. https:/ /doi.org/10.1111/j.1365-2052.2011.02179.x.

Peters, S. O., K. Kizilkaya, D. J. Garrick, R. L. Fernando, J. M. Reecy, R. L. Weaber, G. A. Silver, and M. G. Thomas. 2013. Heritability and Bayesian genome-wide association study of first service conception and pregnancy in Brangus heifers. J. Anim. Sci. 91:605-612. https://doi.org/10.2527/jas.2012-5580.

Petersson, K. J., H. Gustafsson, E. Strandberg, and B. Berglund. 2006. Atypical progesterone profiles and fertility in Swedish dairy cows. J. Dairy Sci. 89:2529-2538. https://doi.org/10.3168/jds.S0022 $-0302(06) 72328-1$.

Pollott, G. E., and M. P. Coffey. 2008. The effect of genetic merit and production systems on dairy cow fertility, measured using progesterone profiles and on-farm recording. J. Dairy Sci. 91:3649-3660. https://doi.org/10.3168/jds.2007-0913.

Pryce, J. E., and R. F. Veerkamp. 2001. The incorporation of fertility indices in genetic improvement programs. Pages 223-226 in Fertility in the High-Producing Dairy Cow. M. G. Diskin, ed. British Society of Animal Science Occasional Publication No. 26, Edinburgh, Scotland.

R Core Team. 2015. R: A language and environment for statistical computing. R Foundation for Statistical Computing, Vienna, Austria. https://www.R-project.org/.

Royal, M. D., A. P. F. Flint, and J. A. Woolliams. 2002. Genetic and phenotypic relationship among endocrine and traditional fertility traits and production traits in Holstein-Friesian dairy cows. J. Dairy Sci. 85:958-967.

Sahana, G., B. Guldbrandtsen, B. Thomsen, L.-E. Holm, F. Panitz, R. F. Brøndum, C. Bendixen, and M. S. Lund. 2014. Genomewide association study using high-density single nucleotide polymorphism arrays and whole-genome sequences for clinical mastitis traits in dairy cattle. J. Dairy Sci. 97:7258-7275. https://doi.org/ $10.3168 /$ jds.2014-8141.

Schmid, M., and J. Bennewitz. 2017. Invited review: Genome-wide association analysis for quantitative traits in livestock-A selective review of statistical models and experimental designs. Arch. Anim. Breed. 60:335-346. https://doi.org/10.5194/aab-60-335-2017.

SNPchimp. 2017. SNPchimp v.3-A multi-species database to disentangle the SNP chip jungle. Gene2Farm. Accessed Oct. 15, 2017. http://bioinformatics.tecnoparco.org/SNPchimp/.

Streit, M., R. Wellmann, F. Reinhardt, G. Thaller, H. P. Piepho, and J. Bennewitz. 2013. Using genome-wide association analysis to characterize environmental sensitivity of milk traits in dairy cattle. G3 (Bethesda) 3:1085-1093. https://doi.org/10.1534/g3 .113 .006536 
Tenghe, A. M. M., A. C. Bouwman, B. Berglund, E. Strandberg, J. Y. Blom, and R. F. Veerkamp. 2015. Estimating genetic parameters for fertility in dairy cows from in-line milk progesterone profiles. J. Dairy Sci. 98:5763-5773. https://doi.org/10.3168/jds.2014-8732.

Tenghe, A. M. M., A. C. Bouwman, B. Berglund, E. Strandberg, D. J. de Koning, and R. F. Veerkamp. 2016. Genome-wide association study for endocrine fertility traits using single nucleotide polymorphism arrays and sequence variants in dairy cattle. J. Dairy Sci. 99:5470-5485. https://doi.org/10.3168/jds.2015-10533.

Teyssèdre, S., M. C. Dupuis, G. Guerin, L. Schibler, J. M. Denoix, J. M. Elsen, and A. Ricard. 2012. Genome-wide association studies for osteochondrosis in French Trotter horses. J. Anim. Sci. 90:45-53. https://doi.org/10.2527/jas.2011-4031.
Veerkamp, R. F., J. K. Oldenbroek, H. J. Van Der Gaast, and J. H. J. Van Der Werf. 2000. Genetic correlation between days until start of luteal activity and milk yield, energy balance, and live weights. J. Dairy Sci. 83:577-583. https://doi.org/10.3168/jds .S0022-0302(00)74917-4.

Zimin, A. V., A. L. Delcher, L. Florea, D. R. Kelley, M. C. Schatz, D. Puiu, F. Hanrahan, G. Pertea, C. P. Van Tassell, T. S. Sonstegard, G. Marçais, M. Roberts, P. Subramanian, J. A. Yorke, and S. L. Salzberg. 2009. A whole-genome assembly for the domestic cow Bos taurus. Genome Biol. 10:R42. https://doi.org/10.1186/gb $-2009-10-4-r 42$. 